\title{
Guest editorial for the special issue on resilient and antifragile ambient systems
}

\author{
Vincenzo De Florio ${ }^{1}$
}

Published online: 12 November 2015

(C) Springer International Publishing Switzerland 2015

This issue of the Journal of Reliable Intelligent Environments is focused on the topic of Resilient and Antifragile Ambient Systems.

The engineering of antifragile computer-based systems is a challenge that, once met, would allow systems to selfevolve and self-improve by learning from accidents and mistakes in a way not dissimilar to that human beings are capable of. Learning how to design and craft antifragile systems is thus an extraordinary challenge whose tackling is likely to reverberate on many a computer engineering field. Computational antifragility for systems and ambients is the main theme of the present issue. The issue includes seven contributions that contribute both practically and theoretically to the state-of-the-art of computational antifragility.

In "On environments as systemic exoskeletons: Crosscutting optimizers and antifragility enablers", Vincenzo De Florio first introduces a general systems classification and then discusses how it may be used to realize intelligent environments able to optimize a systems performance and enable antifragile behaviors. This is exemplified by considering a telemonitoring system developed in the framework of Flemish project Little Sister.

In "Approaches for Engineering Adaptive Systems in Ubiquitous and Pervasive Environments", Mohamed Bakhouya and Jaafar Gaber discuss the main challenges for the engineering of systems able to self-adapt and selfimprove by learning from their interactions with ubiquitous and pervasive environments. The paper also discusses the

Vincenzo De Florio

vincenzo.deflorio@uantwerpen.be;

vincenzo.deflorio@gmail.com

1 MOSAIC Research Group, University of Antwerp and iMinds Institute, Middelheimlaan 1, Building G, Room G1.11, 2020 Antwerp, Belgium usefulness of natural and biological system principles for developing adaptive approaches.

In "Using Bayesian Networks for High Available Cloud based Web Applications", Stefano Marrone discusses Bayesian networks as a language for the optimization of service dependability. This is exemplified in the framework of cloud infrastructures through a model-driven approach. The paper demonstrates how Bayesian networks constitute a natural tool for optimization and learning which in turn are cornerstones to antifragility.

In "Antifragility: systems engineering at its best", Eric Verhulst, Bernhard Sputh, and Pieter Van Schaik discuss the assured reliability and resilience level (ARRL), a novel criterion that allows system components to be reused in a normative way while preserving the safety integrity levels at the system level. ARRL complements the safety integrity level and defines seven levels of which the highest one can be described as the level where the system becomes antifragile. Each level is illustrated and exemplified. It is shown how the features and the general character of ARRL translate into a promising approach for achieving safety across different domains and systems.

In "Modelling Cyber Security for Software Defined Networks That Grow Strong When Exposed to Threats: Analysis and Propositions", Usama Ahmed, Imran Raza, Syed Asad Hussain, Amjad Ali, Muddesar Iqbal, and Xinheng Wang discuss software-defined networks (SDN) as "tools" for the definition of network support against cyber threats. It is the authors' stance that, thanks to SDN, networks may become able to thrive and grow stronger when exposed to overwhelming cyber threats. The authors also review the state-of-the-art of "Security with SDN" and "Security for SDN" and propose a unified model for integrating both approaches.

In "Optimized Congestion Aware and Rate-Adaptive Multimedia Communication for Application in Fragile Envi- 
ronment during Disaster Management and Recovery", Amir Raza Khan, Muddesar Iqbal, Junaid Muzaffar, Amir Nisar, Amjad Ali, Imran Raza, and Syed Asad Hussain present a rate adaptive transcoding and streaming technique that maintains its multimedia streaming quality even when a network grows congested and fragile during disaster management and recovery operations. The proposed solution provides users high-quality audio and video contents even when network resources are limited and fragile.

In "Flexible and Efficient Aggregation Framework for Antifragile Wireless Mesh Networks", the authors Muhammad Shahzad Asif, Muhammad Shafiq, Jin-Ghoo Choi, Muddesar Iqbal, and Azeem Irshad introduce their "Adaptive Aggregation-based Decision Model" (AADM), an intelligent criterion for the choice of which aggregation strategy to adopt in a wireless mesh network. AADM makes it possible for the network to decide when and which type of aggregation is best matching the current scenario. By means of simulations the authors show that AADM reduces control traffic and outperforms existing static approaches in terms of packet loss, throughput, and delay.

This issue also features a regular contribution on a related issue: in "A secure, scalable and versatile multi-layer clientserver architecture for remote intelligent data processing", Gabriele Piantadosi, Stefano Marrone, Mario Sansone, and Carlo Sansone introduce an architecture for remote data processing for smart environments. They propose an architecture for advanced remote data processing in a secure, smart and versatile client-server environment that is capable of integrating pre-existing local software. Feasibility of the approach is investigated in the context of an image-based medical diagnostic environment. 